\title{
Case Report \\ Stenting of Anomalous Left Main Coronary Artery Stenosis in an Adult with a Retroaortic Course
}

\author{
Lanjewar Charan, Santosh Shiradkar, P. G. Kerkar, and Agrawal Ashish \\ Department of Cardiology, King Edward VII Memorial Hospital, Acharya Donde Marg, Parel, Mumbai 400 012, India \\ Correspondence should be addressed to Lanjewar Charan, charanlanjewar@hotmail.com
}

Received 28 February 2011; Accepted 17 May 2011

Academic Editor: Igor Klem

Copyright ( $) 2011$ Lanjewar Charan et al. This is an open access article distributed under the Creative Commons Attribution License, which permits unrestricted use, distribution, and reproduction in any medium, provided the original work is properly cited.

Coronary bypass graft has been the conventional treatment of choice in anomalous left man coronary artery stenosis. We are reporting an interesting case with anomalous left main coronary artery originating from right aortic sinus having retroaortic course complicated by significant atherosclerotic narrowing of the vessel and its percutaneous management.

\section{Case Report}

A 58-year-old diabetic male presented with rest angina followed by syncope. Baseline ECG showed right bundle branch with no significant ST-T changes. However, cardiac troponin was elevated suggesting acute coronary syndrome. Transthoracic Echocardiography was unremarkable with no regional wall motion abnormalities at rest.

Cardiac catheterization with coronary angiography demonstrated anomalous origin of single coronary artery from right sinus of valsalva and dividing into left main and right coronary artery. After getting origin, LMCA had long retroaortic course with significant discrete stenosis in the midstem of it, dividing finally into left anterior descending artery and left circumflex artery. Etiology of coronary occlusion was atherosclerotic plaque and hence was present throughout cardiac cycle and no dynamic cause could be seen. Computerized tomography coronary angiography confirmed the anomalous left main artery origin and its course (Figure 1).

During intervention anomalous coronary was cannulated with $6 \mathrm{~F}$ JR guiding catheter (Brite Tip Cordis company) and lesion was crossed with 0.014 angioplasty guide wire (Boston Scientific) and distally parked in the left circumflex artery. To provide the better support, another 0.014 BMW (Balance Middle Weight, Guidant Corporation) guide wire was kept in left anterior descending artery. Direct stenting was performed successfully with $3.5 \times 12 \mathrm{~mm}$ drug eluting stent (Zotarolimus, Endeavour) at 14 atm (Figure 2). The procedure was uneventful and TIMI 3 flow achieved. The patient is doing very well and is asymptomatic at the recent clinical followup.

\section{Discussion}

Anomalies of the coronary arteries may be found incidentally in $0.3-1 \%$ of healthy individuals. Although coronary artery anomalies are far less common than acquired coronary artery disease, their impact on premature cardiac morbidity and mortality among adults is not trivial. In a study by Eckart et al. of 126 nontraumatic sudden deaths in young adults, cardiac abnormality was found in 64 cases (51\%), with coronary artery abnormalities being the most common cardiac abnormality (39 of 64 patients (61\%)) [1].

Our patient had hemodynamically significant anomalous left main coronary artery characterized by abnormalities of myocardial perfusion.

In the anomalous situation of a single coronary artery, only one coronary artery arises with a single ostium from the aortic trunk. This is an extremely rare congenital anomaly that is seen in only $0.0024 \%$ to $0.044 \%$ of the population. A single coronary artery may follow the pattern of a normal RCA or LCA, divide into two branches with distributions of the RCA and LCA, or have a distribution different from that of the normal coronary arterial tree [2]. 


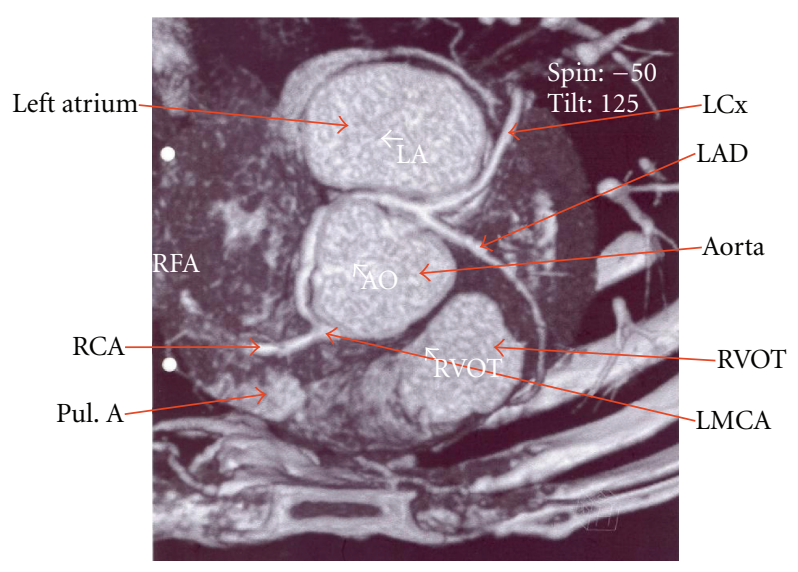

(a)

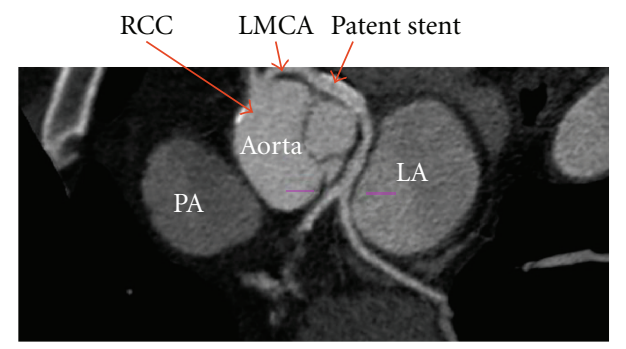

(b)

FIGURE 1: Computerized tomography angiogram. (a) Shows anomalous left main coronary artery (LMCA) arising from the single right coronary cusp and then has the retroaortic course (presenting). Main stem shows significant discrete stenosis. (b) Demonstrates patent stent in anomalous LMCA (after stenting).

Although a single coronary artery may be compatible with a normal life expectancy, patients are at increased risk for sudden death if a major coronary branch crosses between the pulmonary artery and the aorta. The four recognized patterns of an anomalous origin of a coronary artery from the opposite or noncoronary sinus are (a) the RCA arising from the left coronary sinus, (b) the LCA arising from the right coronary sinus, (c) the LCx or LAD artery arising from the right coronary sinus, and (d) the LCA or RCA (or a branch of either artery) arising from the noncoronary sinus, the involved artery may have a high or low takeoff.

In these anomalies, the coronary ostium may be at the normal level, or, moreover, a coronary artery arising from the opposite or noncoronary sinus can take any of the four common courses, depending on the anatomic relationship of the anomalous vessel to the aorta and the pulmonary trunk: (a) interarterial (i.e., between the aorta and the pulmonary artery), (b) retroaortic, (c) prepulmonic, or (d) septal (subpulmonic). It is of great clinical importance which course is taken. Although retroaortic, prepulmonic, and septal (subpulmonic) courses seem to be benign, an interarterial course carries a high risk for sudden cardiac death [3].

Our patient was having retroaortic course, where LMCA was coursing in the dorsal wall of the aorta and, subsequently, between aorta and left atrium.

The LMCA arises from the right sinus of Valsalva as a separate vessel or as a branch of a single coronary artery

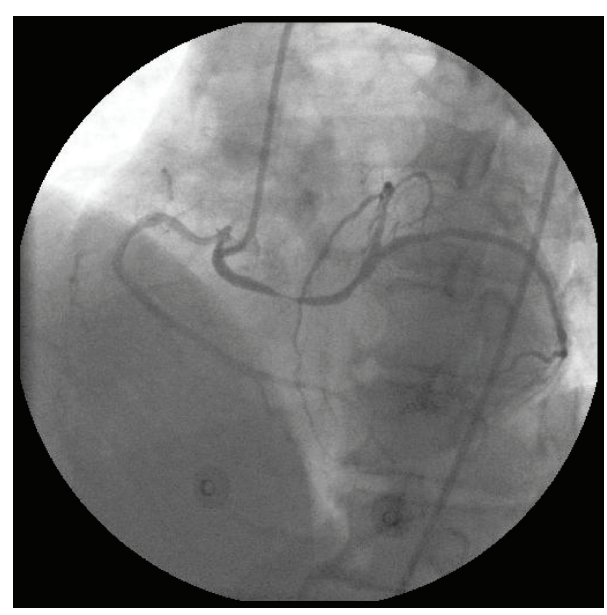

(a)

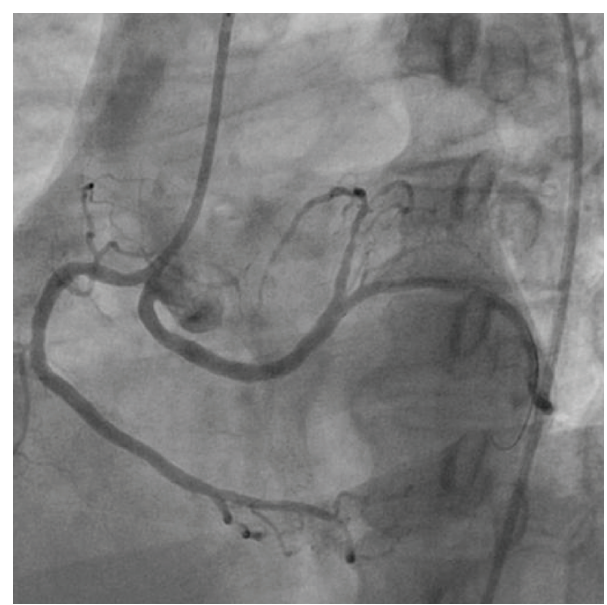

(b)

Figure 2: Coronary angiogram. (a) Anomalous LMCA with significant stenosis and its course. (b) Shows well-deployed stent in LMCA.

in $0.09 \%$ to $0.11 \%$ of angiographic studies, and in up to $75 \%$ of patients with this anomaly the course is interarterial putting them at high risk for sudden cardiac death due to the acute angle of the ostium, the stretch of the intramural segment, and the compression between the commissure of the right and left coronary cusps. However, this anomalous LMCA may also take a retroaortic, prepulmonic, or septal (subpulmonic) course [4].

The Bland-White-Garland syndrome (anomalous origin of the left coronary artery) arising from the pulmonary artery (ALCAPA syndrome) is a rare congenital condition. Eighty percent of affected infants die within 4 months. Survival is critically dependent on the development of collateral circulation. In adulthood, this syndrome is seen with angina, congestive heart failure, mitral regurgitation, and sudden death [5].

We decided to proceed with percutaneous management with stent because of very favorable anatomy of the lesion for PCI and stent placement, minimal operational trauma, and the expected good prognosis. The patient will be closely 
followed up for determination of the benefit from stenting of left main coronary artery.

Guidelines mention that surgical correction is indicated in anamolous left coronary artery arising from right sinus of valsalva and having an interarterial course; however, there are no specific guidelines regarding which subset of patients should be treated percutaneously, but it is reasonable that young patients ( $<35 \mathrm{yrs}$ old) presenting with acute coronary syndrome and diseased anomalous left main coronary artery should be managed with stenting. Coronary artery bypass graft is being increasingly viewed as less favourable option in light of the potential for competitive flow [6]. Patients with incidental diagnosis of anomalous coronaries without symptoms or inducible ischemia should be followed up closely [7].

\section{Conclusion}

Thus, stenting of anomalous left main coronary artery is feasible and a reasonably safe option, and it should be particularly considered in acute coronary syndrome with anomalous LMCA having retroaortic course. However, these patients should be followed up closely for restenosis.

\section{Conflict of Interests}

The authors hereby declare that none of them have any conflict of interests to disclose. This pertains to relationships with pharmaceutical companies, biomedical device manufacturers, or other corporations whose products or services are related to the subject matter of the paper. Such relationships include, but are not limited to, employment by an industrial concern, ownership of stock, membership on a standing advisory council or committee, being on the board of directors, or being publicly associated with the company or its products.

\section{References}

[1] R. E. Eckart, S. L. Scoville, C. L. Campbell et al., "Sudden death in young adults: a 25 -year review of autopsies in military recruits," Annals of Internal Medicine, vol. 141, no. 11, pp. 829834, 2004.

[2] M. A. Greenberg, B. G. Fish, and H. Spindola-Franco, "Congenital anomalies of coronary artery: classification and significance," Radiologic Clinics of North America, vol. 27, pp. 11271146, 1989.

[3] M. D. Cheitlin, C. M. De Castro, and H. A. McAllister, "Sudden death as a complication of anomalous left coronary origin from the anterior sinus of valsalva. A not so minor congenital anomaly," Circulation, vol. 50, no. 4, pp. 780-787, 1974.

[4] N. H. Bunce, C. H. Lorenz, J. Keegan et al., "Coronary artery anomalies: assessment with free-breathing three-dimensional coronary MR angiography," Radiology, vol. 227, no. 1, pp. 201208, 2003.

[5] H. Wesselhoeft, J. S. Fawcett, and A. L. Johnson, "Anomalous origin of the left coronary artery from the pulmonary trunk. Its clinical spectrum, pathology, and pathophysiology, based on a review of 140 cases with seven further cases," Circulation, vol. 38, no. 2, pp. 403-425, 1968.
[6] C. A. Warnes, R. G. Williams, T. M. Bashore et al., "ACC/AHA 2008 Guidelines for the Management of Adults With Congenital Heart Disease," Journal of the American College of Cardiology, vol. 52, no. 23, pp. e1-e121, 2008.

[7] J. A. Davis, F. Cecchin, T. K. Jones, and M. A. Portman, "Major coronary artery anomalies in a pediatric population: incidence and clinical importance," Journal of the American College of Cardiology, vol. 37, no. 2, pp. 593-597, 2001. 


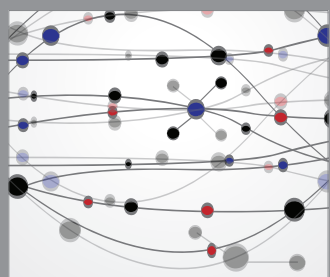

The Scientific World Journal
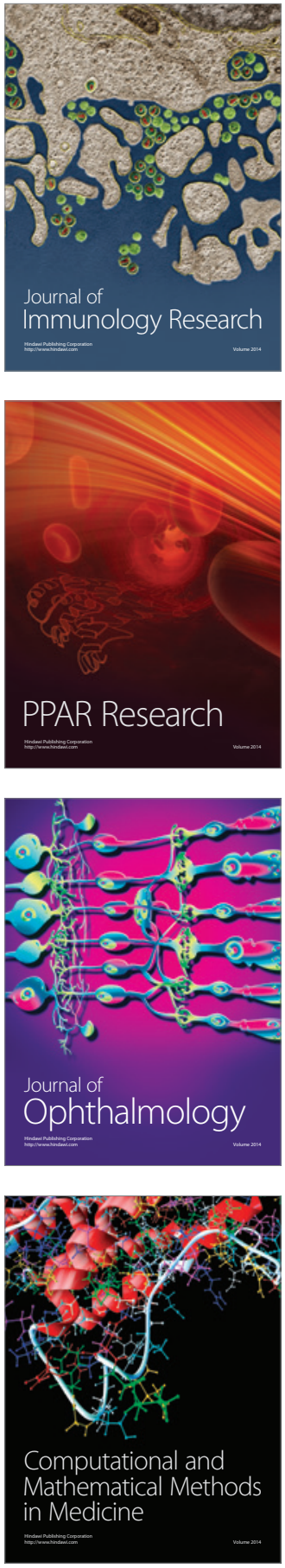

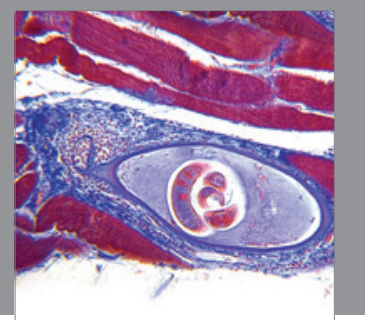

Gastroenterology

Research and Practice
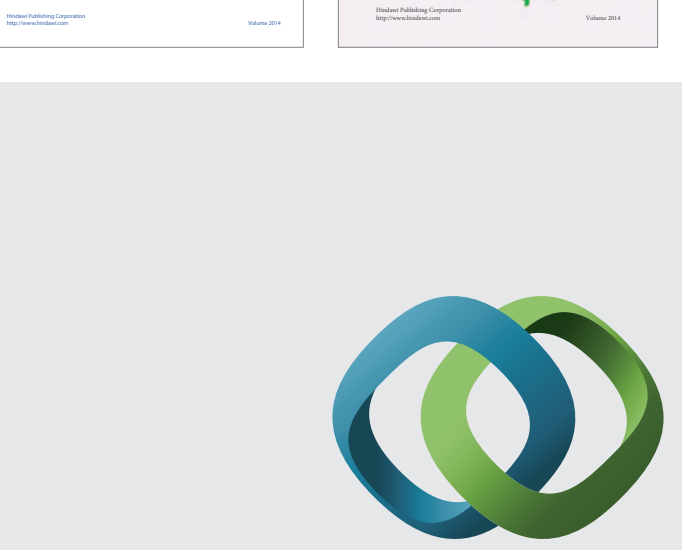

\section{Hindawi}

Submit your manuscripts at

http://www.hindawi.com
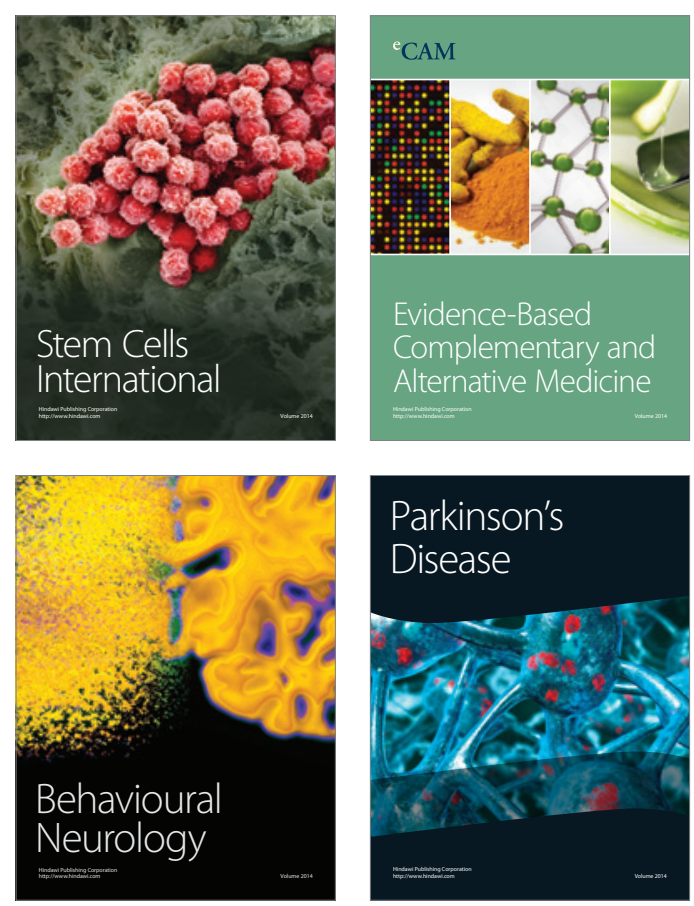

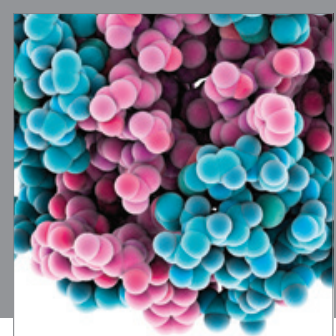

Journal of
Diabetes Research

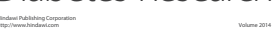

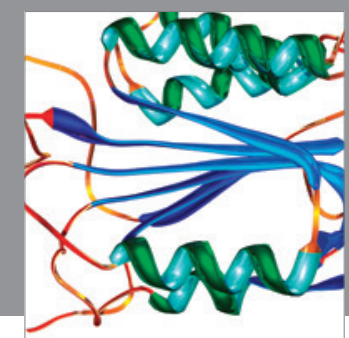

Disease Markers
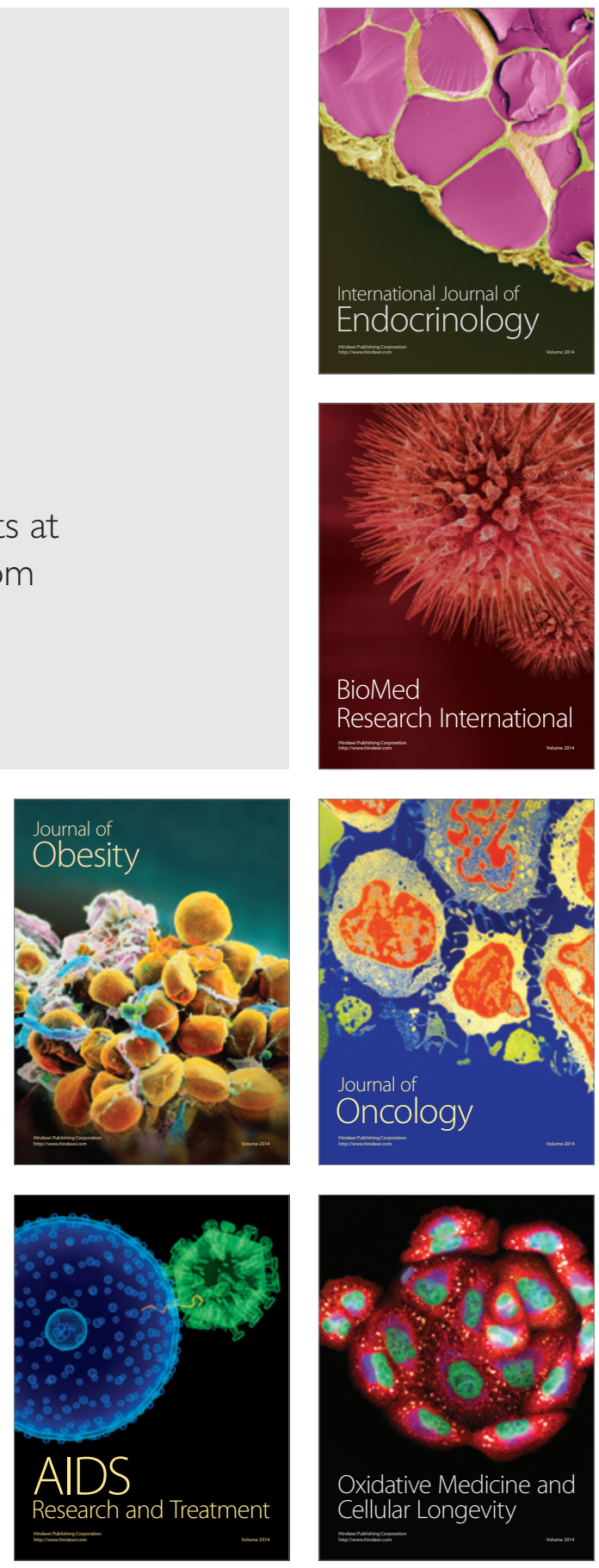